\title{
Quiste óseo esencial: revisión de la literatura a propósito de un caso.
}

\author{
DOI: http//dx.doi.org/10.37315/SOTOCAV202028455126
}

S. PÉREZ-MOTOS, P. GÓMEZ-BARBERO, B. ALASTRUÉ-GINER, J.L. RODRIGO-PÉREZ

SERVICIO DE CIRUGÍA ORTOPÉDICA Y TRAUMATOLOGÍA. HOSPITAL UNIVERSITARIO DOCTOR PESET. VALENCIA.

\section{Resumen.}

El quiste óseo simple (QOS) es una lesión cavitaria típica de edades entre 5 y 15 años que aparece generalmente en las metáfisis de los huesos largos. Se encuentra limitada por una membrana y rellena de líquido, considerándose un pseudoquiste. La lesión se diagnostica como un hallazgo casual en pruebas radiológicas o bien por dolor o fracturas patológicas. Se postulan diferentes etiologías y patogenias de las que se considera más probable el bloqueo sinusoidal o venoso con un aumento de la presión intracameral. El tratamiento tradicional ha sido el curetaje quirúrgico y relleno óseo, apareciendo recientemente nuevas técnicas menos invasivas como la inyección de corticoides y el drenaje del quiste con buenos resultados. En este artículo presentamos un quiste óseo simple de grandes dimensiones en el extremo proximal de fémur tratado mediante punción intracavitaria de corticoides.

Palabras clave: Quiste óseo esencial, niño, diagnóstico diferencial, tratamiento, inyección de corticoides.

\section{Summary.}

Simple bone cyst is a typical cavitated injury which appears between the ages of 5 and 15 and is usually located in long bones' metaphysis. It is limited by a membrane and filled with liquid, being considered a pseudocyst. The cyst is diagnosed as a casual finding in radiological tests as well as for causing pain or pathological fractures.Different aetiologies and pathogenies are postulated, of which sine or venous blockage with increased intracameral pressure is considered to be the most probable. The traditional treatment has been surgical curing and bone filling, with new less invasive techniques such as corticosteroid injection and cyst drainage recently appearing with good results. In this article we present a large simple bone cyst at the proximal end of the femur treated by intracavity puncture of corticosteroids.

\section{Correspondencia:}

Correspondencia:

Sandra Pérez Motos

sandraperezmotos@gmail.com 


\section{Introducción}

El quiste óseo simple (QOS) es una lesión pseudotumoral benigna que generalmente afecta a niños y adolescentes en la segunda década de la vida. Típicamente se localiza en la metáfisis de los huesos largos, adyacente a la fisis, como una cavidad uniloculada o parcialmente separada revestida por una fina membrana que contiene líquido seroso y/o sanguinolento, aunque puede presentarse sin este $^{1-4}$.

Es una lesión relativamente frecuente, que tiende a la curación en la madurez. Durante la infancia muchos son asintomáticos y se descubren incidentalmente durante una exploración radiográfica rutinaria, aunque un $66 \%$ debuta con una fractura patológica con dolor y tumefacción $n^{1,5}$.

El objetivo del trabajo es presentar un caso de QOS localizado en extremo proximal de fémur, discutir aspectos característicos del caso y hacer una breve revisión de la literatura, pues continúa sin existir un consenso sobre el mejor tratamiento ${ }^{6}$.

\section{Caso clínico}

Paciente varón de 12 años de edad que acude a la urgencia de traumatología por coxalgia derecha de 7 días de evolución que se ha exacerbado en las últimas 24 horas. No refiere antecedentes traumáticos ni ningún dato relevante en su historia médica.

A la exploración física presenta balance articular completo con dolor que se incrementa con las rotaciones y la carga axial. No existen signos clínicos ni analíticos de infección activa. Se solicitan radiografías simples de cadera donde se visualiza una lesión lítica sin bordes esclerosos de 97 $\mathrm{mm}$ en la región intertrocantérica y en el cuello del fémur derecho que no invade la fisis. La cortical lateral está adelgazada sin visualizar en la misma ningún trazo de fractura. Se completa el estudio de imagen con una resonancia magnética que confirma el diagnóstico de quiste óseo simple unicameral con septos incompletos sin encontrar interrupciones de la cortical (Fig. 1, 2a y 2b).

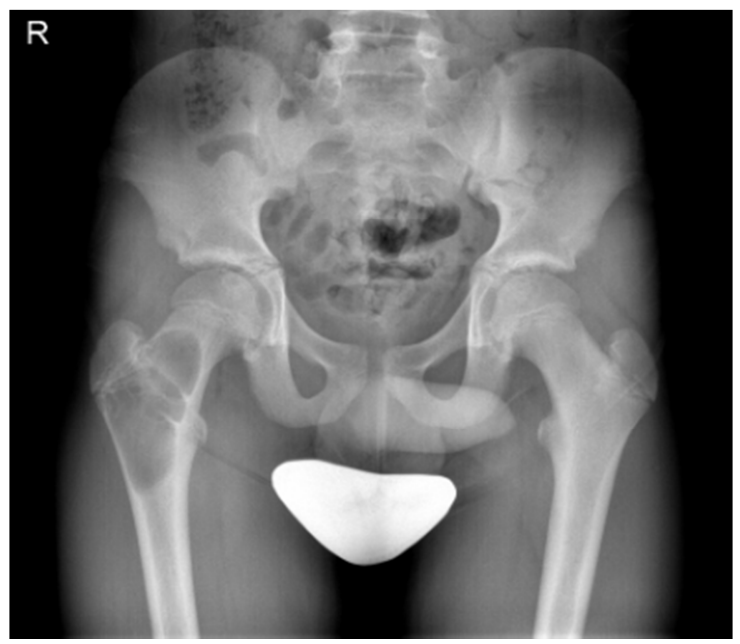

FIGURA 1.Radiografía simple inicial

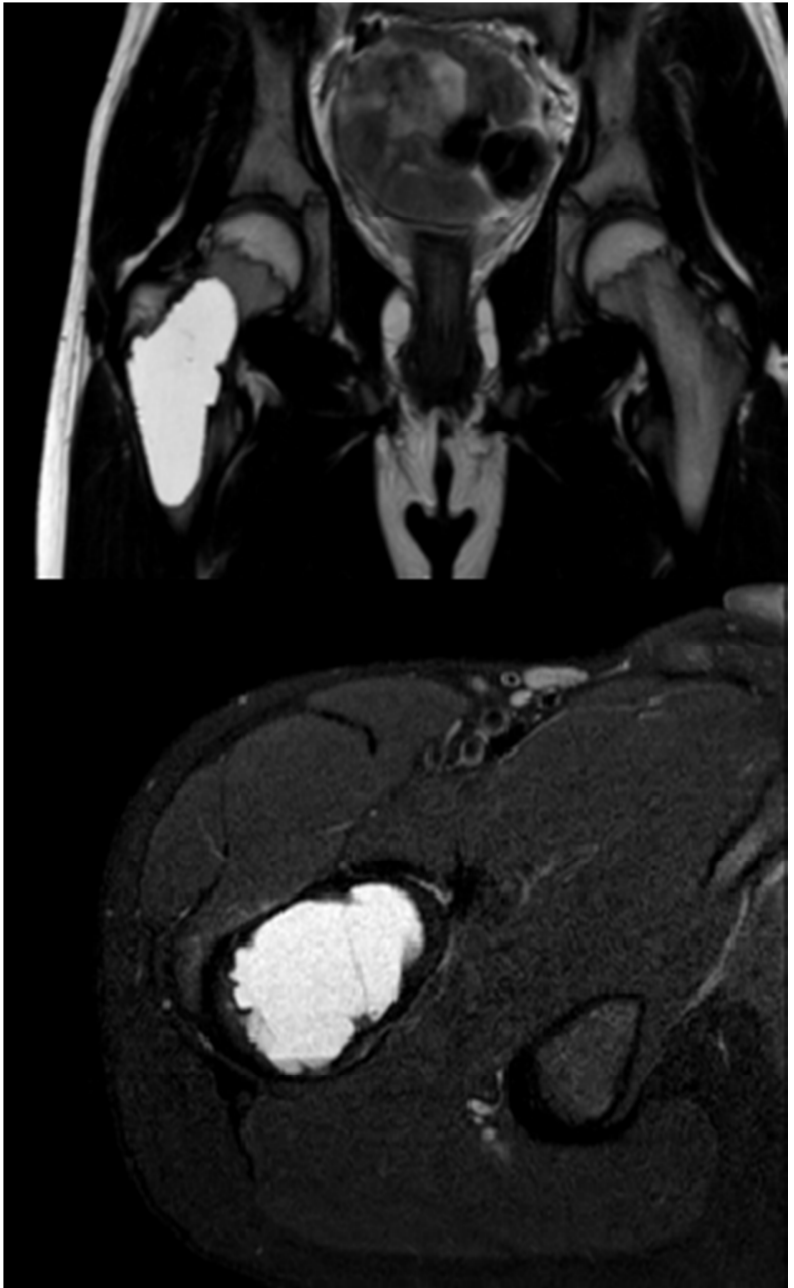

FIGURA 2a y 2b. Resonancia magnética inicial. Corte coronal y axial.

Debido a la clínica, localización, tamaño y al importante adelgazamiento cortical que presenta el fémur derecho se plantea un tratamiento con el fin de evitar una fractura o un trastorno del crecimiento $^{7}$. Bajo condiciones de asepsia y con anestesia general se programa el tratamiento para el QOS unicameral que consiste en la aspiración e infiltración de esteroides. La técnica consiste en la introducción percutánea de 2 trócares a través de los cuales se realiza el aspirado del trasudado, lavado con suero fisiológico e inyección de metilprednisolona.

En el protocolo postoperatorio se mantiene en descarga con muletas y se realizan controles radiográficos cada 2 meses (Fig. 3). En el segundo control radiográfico a los 4 meses de la intervención, aunque existe una leve mejoría clínica y radiológica, no se observan signos evidentes de curación (disminución del tamaño, engrosamiento de la corteza, remodelación ósea, mayor densidad interna con trabeculación), (Fig. 4) por lo que se decide repetir el tratamiento de aspiración, lavado e infiltración de corticoides, encontrando en los controles sucesivos una curación completa del mismo (Fig. 5a, 5b, 6a y 6b). 


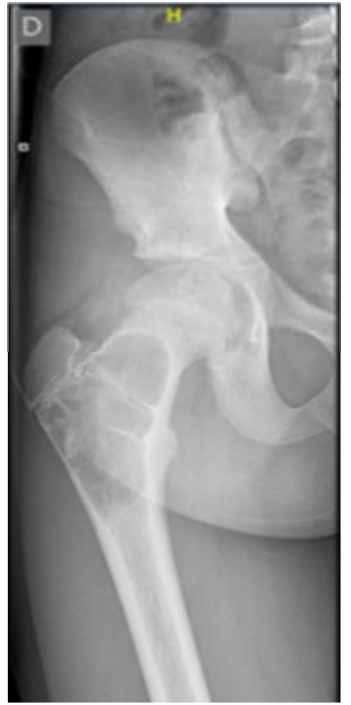

FIGURA 3. 1er Control radiográfico tras el primer tratamiento quirúrgico.

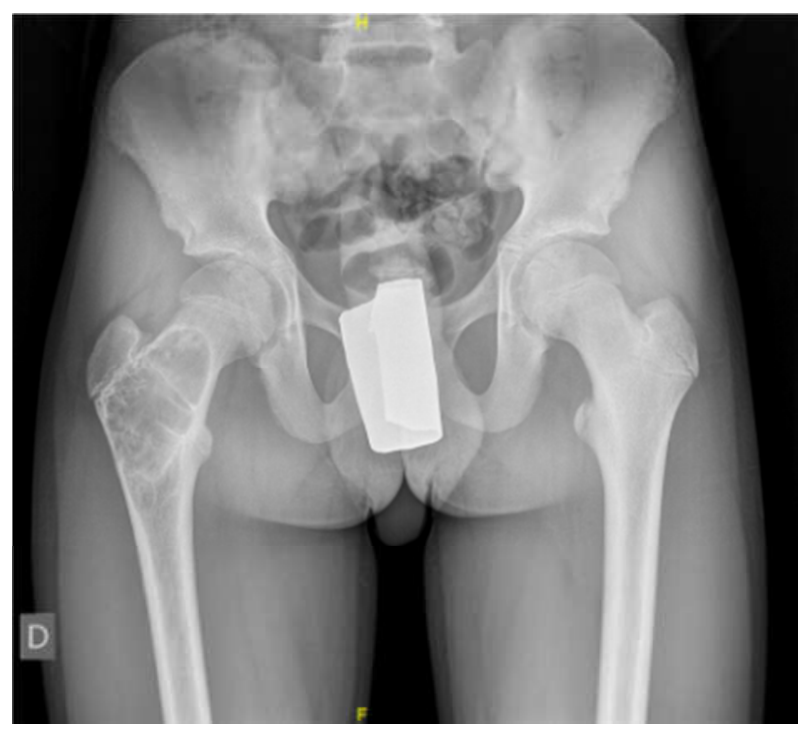

FIGURA 4. Segundo control radiográfico, 4 meses tras la primera intervención.

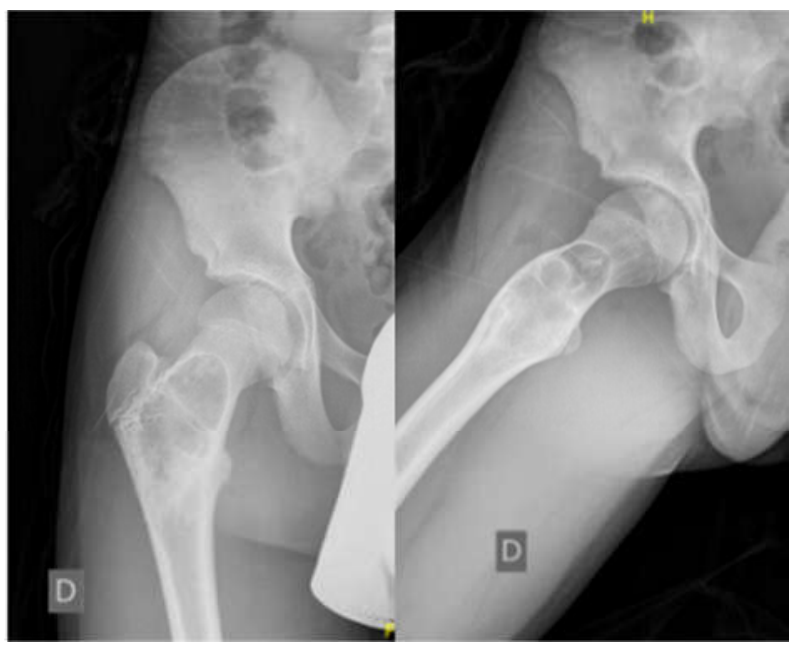

FIGURA 5a y 5b. Control radiográfico a los 2 meses tras repetir el tratamiento con inyección de corticoides. Proyección anteroposterior y axial.

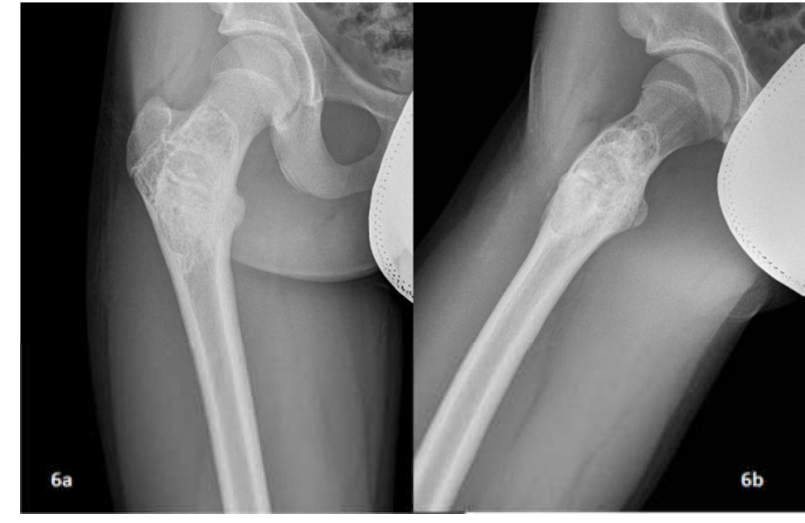

FIGURA 6a y 6b.Último control radiográfico tras 6 meses de la segunda intervención. Proyección anteroposterior y axial.

\section{Discusión}

El QOS es una lesión ósea no neoplásica que se presenta principalmente en niños y adolescentes, y representa el $3 \%$ de todas las lesiones primarias de hueso. Tiene un predominio en varones en una proporción entorno al 2:1 y una incidencia de 1 por 10000 niños por año ${ }^{1,2,4-6,8,9}$.

El primer caso reportado data de 1879. Desde entonces son múltiples los casos publicados con diferentes etiologías que han ido evolucionando desde un fallo en la osificación de la metáfisis durante los periodos de crecimiento rápido, una solución de continuidad en la fisis por una lesión mecánica, una formación a partir de un tejido tumoral, a la existencia de un hematoma intramedular postraumático que provoca una reabsorción osteoclástica. Actualmente, la teoría más aceptada es la descrita en 1977 por Cohen, donde el quiste se forma debido a un bloqueo del drenaje venoso intraóseo con un aumento de la presión intracameral $^{1,3,4,8-10}$.

Por su patogénesis generalmente se localizan en el centro de una metáfisis fértil, junto al cartílago, del que se aleja con el tiempo desplazándose hacia la periferia, aunque en un $2 \%$ de los pacientes afecta a la epífisis a través de la fisis $^{7}$. Las localizaciones más comunes son el tercio proximal del húmero y extremo proximal de fémur, un $85 \%$ de los casos entre las dos localizaciones. Siendo menos frecuente su aparición en tibia y peroné (1-5,8-10. $^{\text {. }}$

Radiológicamente el QOS presenta una imagen muy característica con un área radiolúcida central, la mayoría de veces unilocular, bien delimitada sin margen escleroso. Se sitúa en la metáfisis de los huesos largos, cerca del cartílago y a medida que crece se va localizando en la diáfisis. A diferencia del quiste óseo aneurismático no invade la fisis, siendo muy raro que la atraviese y tampoco produce reacción perióstica, salvo si adelgaza tanto la cortical que produce una fractura patológica. Puede presentar el "signo del fragmento caído" descrito por Roberts cuando existe fractura asociada ${ }^{1,2,4,9}$.

En el examen histopatológico puede identificarse tejido conectivo laxo o fibroso bien vascularizado, con células inflamatorias crónicas, sin componente epitelial en el que 
pueden observarse células gigantes osteoclásticas diseminadas y a veces áreas hemorrágicas con una cortical muy delgada. El líquido contiene altos niveles de citoquinas y prostaglandinas que estimulan la actividad osteoclástica añadido al aumento de la presión responsable de la erosión reabsortiva ${ }^{1,2,9}$.

El quiste óseo es asintomático, y es habitual su diagnóstico casual al realizar una radiografía por cualquier otro motivo. Generalmente tienden a la curación en la madurez pero muchas veces debido a su gran tamaño requieren tratamientos repetidos en la infancia, como en nuestro caso, con intención de disminuir las posibles secuelas derivadas de fracturas o afectación de fisis. En los casos en los que debutan como una fractura patológica, hasta el $66 \%$ de los casos, (se considera la complicación más frecuente de esta lesión y es la causa más frecuente de fractura patológica en niños) se suele tratar de forma conservadora llevando a su curación ${ }^{8,9,11}$.

Suele conseguirse una sospecha diagnóstica con la asociación de clínica, los hallazgos radiográficos, la edad y localización; obteniéndose siempre el diagnóstico definitivo con el análisis histopatológico que requeriría intervención quirúrgica $^{8,11}$. Es de suma importancia realizar un diagnóstico diferencial con otras lesiones metafisarias (Tabla I). Generalmente la RMN (resonancia magnética nuclear) suele permitir la diferenciación de estas lesiones si no ha sido posible mediante el estudio radiográfico simple. Y sólo estará indicado el estudio histopatológico de las lesiones cuando no haya sido posible diferenciación con las pruebas de imagen $^{1,4,9,12}$.

El tratamiento del QOS se realiza generalmente para disminuir el dolor, el riesgo de fractura, de dismetría y de deformidad por retardo de crecimiento longitudinal que puede ocurrir hasta en $14 \%$ de los pacientes. Se reserva el tratamiento expectante sólo en pacientes asintomáticos cercanos a la madurez esquelética sin riesgo de fracturas y con quistes no activos ${ }^{6,8,11}$. Se consideran quistes activos en el momento del diagnóstico aquellos que debutaron con fractura patológica, dolor, los que se encuentran adyacentes a la fisis $(<10 \mathrm{~mm})$ o cuando en el radiografías sucesivas se aprecia un aumento del $25 \%^{5,8,10,11}$.

Ante una fractura patológica suele esperarse a la consolidación de esta para realizar el tratamiento del QOS, existiendo curación espontánea tras la fractura en alrededor de un $8 \%$; si bien es cierto que la consolidación de la fractura provoca la formación de tabiques que dificultan el tratamiento posterior definitivo del quiste, causa entre otras del gran interés en prevenirlas ${ }^{5,8,9,11}$.

Actualmente, la evidencia disponible es insuficiente para determinar el mejor tratamiento ${ }^{6,8}$. La infiltración intralesional de corticoides (IC) es uno de los tratamientos más recomendados, dejando el curetaje con relleno de injerto óseo (preferentemente autólogo) para los quistes que presentan un tamaño importante, corticales muy adelgazadas y los que no responden a IC por presentar una mayor agresividad y una mayor probabilidad de complicaciones. La teoría etiopatogénica sobre el bloqueo del drenaje venoso intraóseo con su consecuente aumento de la presión intracameral justifica el funcionamiento de estas perforaciones de descompresión como tratamiento ${ }^{1,5,10,13}$.

Los porcentajes de curación oscilan en torno al $87 \%$ en caso de curetaje y relleno óseo según el estudio de Campanacci, mientras que son más inconsistentes en caso de IC, variando de un $96 \%$ según Scaglietti $(60 \%$ de curación completa y $36 \%$ de curaciones incompletas), $75 \%$ en el trabajo de Campanacci, $50 \%$ de curación completa en el estudio de Oswaldo (a pesar de obtener $100 \%$ de respuesta en todos los QOS) y $12 \%$ en estudios más actuales como el de Hashemi-Nejad. Oppenheim y Galleno realizaron el primer trabajo comparando ambos tratamientos, con resultados similares para ambas terapias, mientras que Pentimalli - Tudisco y Mylle - Burssens encontraron mejores resultados tras el tratamiento con $\mathrm{IC}^{1,3,5,8,13}$

A causa de la gran discrepancia en estudios han aparecido otras opciones de tratamiento como son las perforaciones y drenaje, las inyecciones percutáneas de médula ósea, el relleno con cubos de hidroxiapatita y la resección total o subtotal $^{4,13}$.

El tratamiento mediante punción con metilprednisolona fue publicado por primera vez en 1974 por Scaglietti y posteriormente ha sido corroborado por diferentes autores, con un alto porcentaje de resultados favorables ${ }^{1,4,10}$. Existen dos mecanismos diferentes que justifican que la punción con corticoides funcione; por un lado el mecanismo de la punción en sí disminuyendo la presión, apoyando la hipótesis del bloqueo sinusoidal y por otro lado por el efecto del corticoide en su interior, gracias a su acción en los lisosomas y su fenómeno frente a la autoinmunidad que ayuda a la destrucción sobre el tejido conjuntivo de la pared quística $^{1,3,4,10,11}$.

Se recomienda el uso de dos trócares y de contraste radiográfico para observar si el relleno de la cavidad quística es completo o si hay comunicación entre las diferentes cavidades que permita el relleno total ${ }^{1,3,8,10}$. En nuestro caso no fue preciso por haber realizado previamente una RMN que aportó información sobre la presencia de tabiques en el quiste ${ }^{4}$.

Si el quiste es de gran tamaño, para disminuir el riesgo de fractura se puede mantener en descarga hasta observar la osificación de la cavidad quística ${ }^{3,12}$. En nuestro caso se mantuvo al paciente tras la primera intervención en descarga durante las primeras 8 semanas y carga parcial hasta el momento en el cual se repitió el tratamiento. Mientras que tras la segunda intervención, dado la disminución del tamaño se optó por 4 semanas sin apoyo completo y 2 más con apoyo parcial. A los 7 meses tras el último lavado y relleno del quiste se autorizó la actividad deportiva.

Es recomendable realizar revisiones periódicas, pudiéndose practicar nuevas infiltraciones si no hay respuesta o si es insuficiente. En diferentes estudios se realizan las infiltraciones al mes y a los 2 meses o 3 meses, aunque en las recomendaciones más actuales no se aconseja el intervalo menor de 3 meses y se recomienda el 
seguimiento hasta la maduración ósea por el riesgo de recidivas $^{3-5,8,10,11,13}$. En nuestro paciente han sido precisas 2 infiltraciones para la visualización de relleno del quiste, la segunda a los 4 meses de la primera intervención. Actualmente se mantiene el seguimiento del paciente en consultas externas de nuestro servicio.

Los fallos de curación se asocian a quistes multilobulados y de manera inconstante en diferentes estudios se ha asociado también a la actividad del quiste, a la proximidad a la fisis, a la edad y al sexo del paciente, siendo más frecuente la recidiva en varones jóvenes ${ }^{3,5,8,10,11}$.

\section{Conclusión}

Después de revisar la bibliografía, en nuestra experiencia el tratamiento con lavado del quiste e inyección intracameral de corticoides representa una alternativa de baja agresividad factible para el tratamiento del quiste óseo esencial con buenos resultados, que permite repetir las infiltraciones y un cambio a un tratamiento más invasivo si existiese un fallo de curación.

Tabla 1. Diagnóstico diferencial del quiste óseo simple $e^{1,4,9,12}$

\begin{tabular}{|c|c|c|}
\hline Posibles diagnósticos diferenciales & Localización más frecuente & Otras características \\
\hline Quiste óseo esencial & $\begin{array}{l}\text { Metáfisis, excepcionalmente diáfisis. Huesos } \\
\text { largos. Fémur, húmero y tibia. }\end{array}$ & $\begin{array}{l}\text { Localización central, densidad líquida y cortica } \\
\text { adelgazada. } \\
\text { Signo del fragmento desprendido } \\
\text { (patognomónico), signo de la escotilla en } R x \text {. } \\
\text { No supera fisis ni desarrolla reacción perióstice }\end{array}$ \\
\hline Quiste óseo aneurismático & Metáfisis-diáfisis. Fémur, tibia y vértebras. & $\begin{array}{l}\text { Forma excéntrica, pudiéndose desplazar } \\
\text { centralmente. } \\
\text { Presenta múltiples tabiques y niveles líquido- } \\
\text { líquido (distribución en estallido o burbujas } \\
\text { de jabón), desarrolla inflamación en el hueso } \\
\text { circundante. } \\
\text { Puede superar la fisis y presentar reacción } \\
\text { perióstica. }\end{array}$ \\
\hline Displasia fibrosa & $\begin{array}{l}\text { Metáfisis, Confunde en su forma solitaria. } \\
\text { Fémur, húmero, mandíbula. }\end{array}$ & $\begin{array}{l}\text { Límites menos claros,imagen en vidrio } \\
\text { deslustrado por las trabéculas finas (muy } \\
\text { característica), densidad sólida. Pueden } \\
\text { existir deformidades angulares. Deformidad } \\
\text { en cayado del pastor (fémur). }\end{array}$ \\
\hline Fibroma no osificante o defecto fibroso cortical & Metáfisis. Huesos largos. Fémur y tibia. & $\begin{array}{l}\text { Lesión más frecuente en esta edad. Foco de } \\
\text { inicio cortical, bajo inserción de un tendón o } \\
\text { ligamento. } \\
\text { Lesión irregular, línea de esclerosis rodeando } \\
\text { la lesión, polilobulada con tabiques ( racimo } \\
\text { de uvas). Se separa de la fisis con el } \\
\text { crecimiento. }\end{array}$ \\
\hline Granuloma eosinófilo & $\begin{array}{l}\text { Diáfisis. Cráneo, región proximal de fémur, } \\
\text { vértebras dorsales. }\end{array}$ & $\begin{array}{l}\text { Dolor elevado. } \\
\text { Lesión sólida osteolítica de menor tamaño, } \\
\text { límites menos claros e irregulares. Reacción } \\
\text { perióstica habitual. } \\
\text { Se observan varias lesiones en la forma } \\
\text { múltiple (Histiocitosis de Langerhans). }\end{array}$ \\
\hline
\end{tabular}




\begin{tabular}{|l|l|l|}
\hline Tumor de células grandes & $\begin{array}{l}\text { Epífisis cerradas. En ocasiones en metáfisis } \\
\text { (cuando aparecen en esqueleto inmaduro). } \\
\text { Rodilla. }\end{array}$ & $\begin{array}{l}\text { Más frecuente en adultos y sexo femenino. } \\
\text { Se presenta como una zona radiolucente } \\
\text { expansiva (charco en arena), excéntrica y } \\
\text { subcondral. Adelgaza cortical, no produce } \\
\text { reacción perióstica. }\end{array}$ \\
\hline Encondroma & $\begin{array}{l}\text { Diáfisis. Huesos tubulares cortos } \\
\text { (localización menos frecuente del QOS) }\end{array}$ & $\begin{array}{l}\text { Más frecuente en adultos. Lesiones } \\
\text { puntiformes ( calcificaciones dentro de tumor } \\
\text { cartilaginoso). } \\
\text { Lesión lítica, de menor tamaño que expande } \\
\text { cortical. }\end{array}$ \\
\hline
\end{tabular}

\section{Bibliografía}

1. Revenga Giertych C, Bulo Concellón MP, Carrascosa Cerquero A, Gutiérrez Nogueroles M, Baudet Carrillo M. Tratamiento quirúrgico del quiste óseo esencial. Rev. S. And. Traum. y Ort 2001; 21(2): 212-9.

2. Chrcanovic BR, López Alvarenga R, Freire-Maia B. Quiste óseo simple: Reporte de un caso y revisión de la literatura. Avances en odontoestomatología 2011; 27 (4):207-13.

3. Gasco J, Del Pino JM, Gomar Sancho F. Quiste óseo esencial. Tratamiento mediante inyección intracavitaria de acetato de metilprednisolona. Rev Esp Cir Osteoart 1984; 19 (111):149-64.

4. Docquier L, Delloye C. Quiste óseo esencial. EMC- Técnicas quirúrgicas en ortopedia y traumatología 2011; 44(2):1-9.

5. Alonso Hernández J, Abril Martín JC, Epeldegui Torre T, Gil de Rozas M, Carrillo Dutor R. Tratamiento del quiste óseo simple en la población infantil. Rev Esp Cir Ortop y Traumatol 2003; 47:48-54.

6. Zhao JG, Wang J, Huang WJ, Zhang $\mathbf{P}$, Ding $\mathbf{N}$ et al. Interventions for treating simple bone cysts in the long bones of children. Cochrane Database of Systematic Reviews 2017; 2.

7. Miranda Gorozarri C, Montero Díaz M, Abril JC, Azorín Cuadrillero D. Quiste óseo simple. En: Ortiz Cruz E.J., Ramos Pascua L.R., directores. Curso de Tumores SECOT. III Edición. 2020.

8. Pellicer García V, Ortego Sanz J, Salom Taverner M, Miranda Casas L, Lorente Moltó F. et al. Evaluación clínica-radiográfica de la efectividad de las infiltraciones percutáneas de acetato de metilprednisolona en el tratamiento de los quistes óseos esenciales. Rev Esp Cir Osteoart 2011; 46(245):18-25.

9. Obrero Gaitán D, Combalía Aleu A, Ramos Pascua L. Tumores óseos y lesiones pseudotumorales: tumores de la serie condral y otras. Metástasis. Lesiones paratumorales. En: Delgado Martinez A., Marco Martinez F., Guerado Parra E., García Alonso M., Marchal Corrales J.A., coordinadores. Curso COT: Programa de Formación en Cirugía Ortopédica y Traumatología. $5^{\circ}$ Edición. 2019.

10. Suárez-Suárez M.A., Maestro Fernández A., Murcia Mazón A., Guerra García C., Acebal Cortina G. et al. Quiste óseo simple: estudio comparativo de diferentes métodos de tratamiento. Rev Esp Cir Osteoart 1998; 33 (194): 71-8.

11. Del Pino Ortiz JM, Sanchis Alfonso V, Gascó J, Gomar Sancho F. Quiste óseo esencial. Resultados del tratamiento mediante punción intracavitaria con corticoides. Rev Esp Cir Osteoart 1992; 27:15-21.

12. Tejada Gallego J, Del Castillo Codes M, Martinez Caballero I. Displasias óseas. En: Delgado Martinez A., Marco Martinez F., Guerado Parra E., García Alonso M., Marchal Corrales J.A., coordinadores. Curso COT: Programa de Formación en Cirugía Ortopédica y Traumatología. $5^{\circ}$ Edición. 2019.

13. Cuenca Espiérrez J, Herrera Rodríguez A, Gil Albarova J, Martínez Martín AA, Bregante Baquero J. El quiste óseo esencial en la infancia. Estudio retrospectivo de 15 casos y revisión de la literatura. Cir Pediatr 2002; 15:68-72. 